\title{
DINÂMICA DE ESPÉCIES ARBÓREAS EM FLORESTA ESTACIONAL DECIDUAL RIPÁRIA NO NOROESTE DO RIO GRANDE DO SUL
}

\author{
DYNAMICS OF ARBOREAL SPECIES IN RIPARY DECIDUAL SEASONAL FOREST IN THE \\ NORTHWEST OF RIO GRANDE DO SUL
}

\author{
Camila Andrzejewski ${ }^{1}$, Solon Jonas Longhi ${ }^{2}$, Rafael Marian Callegaro ${ }^{3}$, Elke Lima dos Santos ${ }^{4}$ \\ 1,2,4 Universidade Federal de Santa Maria, Santa Maria, RS, Brasil - camila_andrzejewski@hotmail.com, \\ longhi.solon@gmail.com \& elke.lima@hotmail.com
}

${ }^{3}$ Universidade Federal do Pampa, São Gabriel, RS, Brasil - rafaelcallegaro@unipampa.edu.br

\begin{abstract}
RESUMO
O objetivo deste trabalho foi analisar mudanças temporais em nível de comunidade e de população em dois fragmentos de Floresta Estacional Decidual ripária, na região do Alto Uruguai, no Rio Grande do Sul, Brasil. Foram instaladas sistematicamente 36 parcelas, distribuídas em faixas perpendiculares à margem do rio. 0 desenho amostral foi aplicado nos dois fragmentos, cada um contendo 18 parcelas com dimensões de $10 \mathrm{~m} \times 20 \mathrm{~m}$, onde foram amostrados os indivíduos com diâmetro à altura do peito (DAP) igual ou maior a $5 \mathrm{~cm}$. A vegetação foi inventariada nos anos de 2015 a 2019. As curvas de rarefação indicaram que não houve diferença de riqueza entre as ocasiões. A análise da dinâmica mostrou taxa de mudança em densidade de indivíduos positiva no primeiro ano de monitoramento, quando também ocorreu a maior variação líquida em área basal. Nos últimos dois anos a vegetação apresentou taxas de mudança negativas para densidade e dominância. Os resultados evidenciaram flutuações pouco expressivas na densidade, no número de espécies raras, na proporção de indivíduos e de espécies de cada grupo ecológico, bem como da distribuição diamétrica da comunidade e das espécies de maior valor de cobertura. As espécies que tiveram maior valor de cobertura foram Matayba elaeagnoides Radlk., Campomanesia xanthocarpa (Mart.) O.Berg, Gymnanthes klotzschiana Müll.Arg. e Actinostemon concolor (Spreng.) Müll.Arg., indicando que as mesmas são mais representativas nos fragmentos.
\end{abstract}

PALAVRAS-CHAVE: Fitossociologia, Mata ciliar, Mudança temporal, Taxa de mudança.

\section{ABSTRACT}

The objective of this work was to analyze temporal changes at the community and population level in two fragments of riparian seasonal deciduous forest, in the Alto Uruguai region, in the Rio Grande do Sul, Brazil. 36 plots were systematically installed, distributed in perpendicular strips to the riverbank. The sample design was applied to the two fragments, each containing 18 plots with dimensions of $10 \mathrm{~m} \mathrm{x} 20 \mathrm{~m}$, where individuals with a diameter at chest height (DBH) equal to or greater than $5 \mathrm{~cm}$ were sampled. The vegetation was inventoried in the years 2015 to 2019 . The rarefaction curves indicated that there was no difference in wealth between the occasions. The dynamics analysis showed a positive change rate in the density of individuals in the first year of monitoring when there was also the greatest net variation in the basal area. Over the past two years, vegetation has shown negative change rates for density and dominance. The results showed insignificant fluctuations in density, in the number of rare species, in the individuals proportion and species in each ecological group, as well as in the diametric distribution of the community and the species with the highest coverage value. The species that had the highest coverage value were Matayba elaeagnoides Radlk., Campomanesia xanthocarpa (Mart.) O.Berg, Gymnanthes klotzschiana Müll.Arg. and Actinostemon concolor (Spreng.) Müll.Arg., indicating that they are more representative in the fragments.

KEYWORDS: Change rate, Phytosociology, Riparian forest, Temporal change. 


\section{INTRODUÇÃO}

Uma das maiores preocupações para a conservação da biodiversidade é a contenção das intervenções humanas que ocasionam a fragmentação dos ecossistemas e consequentemente a perda ou redução de habitats (VIANA \& PINHEIRO, 1998). Grande parte das formações florestais no Rio Grande do Sul está representada por pequenas manchas florestais localizadas ao longo de cursos d'água, podendo ser denominadas de fragmentos florestais ripários, que refletem alterações ecológicas atuantes nesses ecossistemas.

As florestas ripárias são zonas de transição entre ecossistemas terrestres e aquáticos e desempenham serviços ecossistêmicos como proteção da biodiversidade terrestre e dinâmica de estoques de carbono (GUNDERSEN et al., 2010). Essas formações florestais foram reduzidas a pequenos fragmentos pela ação antrópica, relacionadas à expansão desordenada das fronteiras agrícolas (RODRIGUES \& GANDOLFI, 2004), com alto grau de perturbação e, de acordo com Venzke \& Martins (2013), localizam-se principalmente em ambientes de topografia acidentada, áreas pedregosas sem aptidão agrícola e fundo das propriedades.

Os fragmentos ripários ocupam áreas dinâmicas da paisagem, apresentando espécies com características particulares para melhor adaptação aos distúrbios (NAIMAN \& DÉCAMPS, 1997; SARAIVA, 2011). Além disso, apresentam variação espaço-temporal em virtude da heterogeneidade ambiental, onde vários fatores são potencialmente determinantes da composição e estrutura de espécies em pequenas formações florestais, porém, poucas pesquisas são realizadas nesses locais.

Diante das mudanças das condições ambientais e da vegetação nas áreas ripárias sujeitas a inundações, fica intrínseca a necessidade de monitorar as variações ao longo do tempo para inferir com maior precisão sobre os efeitos da fragmentação. Higuchi et al. (2008) ressaltaram que as respostas à fragmentação podem ser um processo lento capaz de ocorrer durante longos períodos. Para Fontes \& Walter (2011), o monitoramento da dinâmica permite compreender a complexidade dos processos ecológicos e as interações interespecíficas.

As pesquisas sobre análise da dinâmica da vegetação podem subsidiar o entendimento da sucessão florestal. Contudo, este tema não foi abordado em trabalhos publicados englobando áreas na região noroeste do Rio Grande do Sul, local caracterizado por Floresta Estacional Decidual. Assim, o presente estudo teve como objetivo analisar as mudanças em parâmetros de estrutura e de crescimento, bem como descrever as flutuações temporais de dinâmica detectadas em populações de espécies ocorrentes no estrato arbóreo em fragmentos de Floresta Estacional Decidual ripária.

\section{MATERIAL E MÉTODOS}

\section{Caracterização da área}

O presente estudo foi realizado em fragmentos florestais ripários, pertencentes à região fitoecológica da Floresta Estacional Decidual, incluindo as formações Aluvial e Submontana (IBGE, 2012). Selecionou-se para o monitoramento dois fragmentos situados às margens do rio ljuí, no município de Guarani das Missões, Rio Grande do Sul, com maior extensão em área e sem influência de barragens, comuns na região. Os fragmentos localizam-se nas comunidades Linha do Mel (28 $15^{\prime} 30,70^{\prime \prime} \mathrm{S}$ e $54^{\circ} 34^{\prime} 25,77^{\prime \prime}$ O), com 6 ha, e Pinheiro Machado

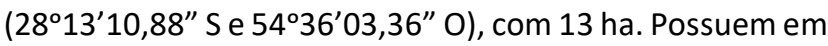
suas adjacências áreas de cultivo agrícola ou pastejo de pecuária leiteira, limitando-se a oeste com o rio.

A altitude média das áreas é de $170 \mathrm{~m}$ e o relevo é suave ondulado, onde predominam, segundo Streck et al. (2008), solos profundos do tipo Latossolo Vermelho Distroférrico. O clima da região é do tipo Cfa (subtropical úmido, com verão quente), de acordo com a classificação climática de Köppen (ALVARES et al., 2013). A precipitação pluviométrica anual é de aproximadamente $1800 \mathrm{~mm}$, ocorrendo chuvas bem distribuídas durante o ano, com temperatura média anual de $21^{\circ} \mathrm{C}$ (WREGE et al., 2011).

\section{Coleta de dados}

Para amostragem da vegetação foram instaladas 18 parcelas por fragmento, com dimensões de $10 \mathrm{~m}$ x $20 \mathrm{~m}$ $\left(200 \mathrm{~m}^{2}\right)$, no ano de 2015 . As parcelas foram distribuídas na vegetação em faixas sistemáticas, distanciadas $40 \mathrm{~m}$ entre si e perpendiculares ao curso do rio. Nas faixas foi mantida a distância de $20 \mathrm{~m}$ entre o fim de uma parcela e o início de outra (Figura 1).

O estrato arbóreo foi inventariado em todas as parcelas, onde incluíram-se todos os indivíduos com diâmetro medido a 1,30 de altura do solo $(D A P) \geq 5 \mathrm{~cm}$. Os indivíduos foram identificados e tiveram a circunferência à altura do peito (CAP), para posterior cálculo do DAP, e a altura total $(\mathrm{H})$ medidas.

A coleta de dados da vegetação foi realizada nos meses de fevereiro de cada ano (2015 a 2019). Os indivíduos amostrados foram etiquetados com plaqueta metálica 
contendo código de identificação.

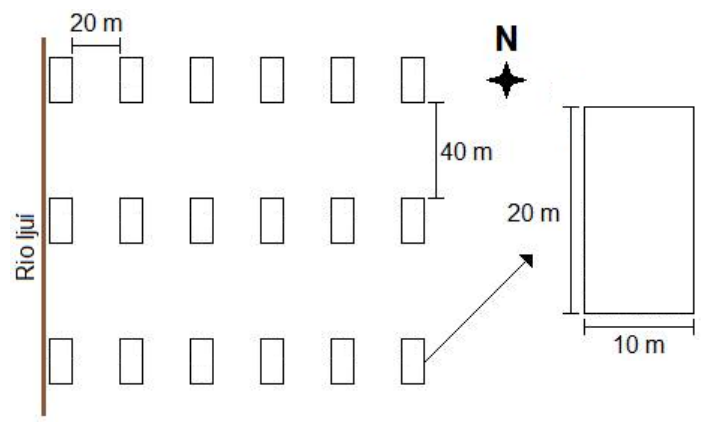

Figura 1. Desenho amostral das parcelas do estrato arbóreo. Guarani das Missões, RS, Brasil. 2020. Adaptado de Andrzejewski (2016).

Foi coletado material botânico dos indivíduos não identificados no local para identificação através de bibliografias junto ao Herbário do Departamento de Ciências Florestais da Universidade Federal de Santa Maria (HDCF). A nomenclatura das espécies foi conferida no banco de dados Flora do Brasil 2020 (FORZZA et al., 2020) e a delimitação das famílias seguiu o sistema de classificação Angiosperm Phylogeny Group IV (BYNG et al., 2016).

\section{Análise dos dados}

Comparou-se a riqueza na primeira medição (2015) e na última medição (2019) através de curvas de rarefação. Para isto, foram utilizadas matrizes constituídas por espécies, nas linhas, e por abundância de cada espécie, nas colunas. A análise foi realizada pelo programa PAST (HAMMER et al., 2001).

Foram contabilizados o número de indivíduos, para posterior cálculo da densidade, e a área basal por espécie para cada ocasião de monitoramento (FELFILI \& REZENDE, 2003; CALLEGARO et al., 2014). Neste estudo, a dominância será tratada como área basal por hectare $\left(\mathrm{m}^{2} / \mathrm{ha}\right)$.

As espécies foram classificadas em grupos ecológicos, de acordo com Swaine \& Whitmore (1988) e Oliveira-Filho et al. (1994). Assim, as espécies que necessitam de luminosidade solar plena em processos de regeneração, crescimento, desenvolvimento e sobrevivência foram classificadas em pioneira; em clímax exigente de luz foram classificadas as espécies que podem germinar sob sombreamento, mas as plantas jovens necessitam de luz abundante para crescer e atingir o dossel e a maturidade; e por fim em clímax tolerantes à sombra, que podem se desenvolver sob sombreamento, podendo atingir a maturidade no dossel ou antes de alcançar o dossel.
Para verificar se houve diferença de densidade de indivíduos e de espécies ao longo do tempo dentro do mesmo grupo ecológico ou em cada ano entre os grupos ecológicos foi utilizado o teste não-paramétrico de KruskalWallis, seguida do teste de Dunn para as comparações quando o teste não-paramétrico indicou diferenças significativas, por meio do programa BioEstat 5.3 (AYRES et al., 2007). Foram aplicados testes não-paramétricos devido ao não atendimento dos pressupostos de normalidade dos resíduos (Teste de Shapiro-Wilk) e homogeneidade das variâncias (Teste de Bartlet).

Para analisar a variação de atributos da estrutura e da dinâmica em diferentes intervalos de tempo (anual, bienal e quadrienal), foram calculados descritores em nível de comunidade baseados na densidade de indivíduos (D) e na área basal ( $A B)$, utilizados por Higuchi et al. (2008) e Oliveira et al. (2014): densidade no início do intervalo de tempo ( $\left.D_{\text {inicial }}\right)$; de sobreviventes ( $\left.D_{\text {sobreviventes }}\right)$; de recrutas ( $\left.D_{\text {recrutas }}\right)$; de árvores mortas ( $\left.D_{\text {mortas }}\right)$; e no final do intervalo de tempo ( $\left.D_{\text {final }}\right)$; bem como taxa de mudança (TMdensidade), taxa de mortalidade (TMort) e taxa de recrutamento (TR).

Além destes, foram calculados os descritores área basal inicial ( $\left.A B_{\text {inicial }}\right)$, aumento em área basal ( $\left.A B_{\text {aumento }}\right)$, decréscimo em área basal ( $A B$ decréscimo), área basal de recrutas $\left(A B_{\text {recrutas }}\right)$, área basal de mortas ( $A B$ mortas), área basal ao final do período de monitoramento $\left(A B_{\text {final }}\right)$, taxa de mudança $\left(T M_{A B}\right)$, ganho em área basal $\left(G_{A B}\right)$ e perda em área basal $\left(\mathrm{P}_{\mathrm{AB}}\right)$. As taxas de mudança de densidade (Equação 1) e de área basal (Equação 2), as taxas de mortalidade (Equação 3) e de recrutamento (Equação 4), bem como o ganho (Equação 5) e perda (Equação 6) foram calculados conforme Korning \& Balslev (1994) e Sheil \& May (1996).

$$
\begin{gathered}
T_{D}=\left(\left(\frac{D_{\text {final }}}{D_{\text {inicial }}}\right)-1\right) \times 100 \\
T_{A B}=\left(\left(\frac{A B_{\text {final }}}{A B_{\text {inicial }}}\right)-1\right) \times 100 \\
T M o r t=\left(1-\left(\frac{D_{\text {inicial }}-D_{\text {mortas }}}{D_{\text {inicial }}}\right)\right) \times 100 \\
P_{A B}=\left(1-\left(\frac{\left(A B_{\text {inicial }}-\left(A B_{\text {mortas }}+A B_{\text {decréscimo }}\right)\right)}{A B_{\text {inicial }}}\right)\right) \times 100 \\
\left.G_{A B}=\left(1-\left(1-\frac{\left(A B_{\text {recrutas }}+A B_{\text {aumento }}\right)}{A B_{\text {final }}}\right)\right)\right) \times 100
\end{gathered}
$$

Em que: $\mathrm{TM}=$ taxa de mudança $(\%) ; \mathrm{D}=$ densidade (indivíduos $/ \mathrm{ha}) ; A B=$ área basal $\left(\mathrm{m}^{2} / \mathrm{ha}\right) ;$ TMort $=$ taxa de mortalidade (\%); TR = taxa de recrutamento (\%); $\mathrm{G}=$ ganho em área basal (\%); e $\mathrm{P}=$ perda em área basal (\%). 
A análise de estrutura diamétrica foi realizada para a comunidade e para as quatro espécies de maior valor de cobertura em 2015 e em 2019 (Valor de cobertura = densidade relativa + dominância relativa). Os números de classes diamétricas e os intervalos de classe foram calculados conforme a Equação 7 e a Equação 8, respectivamente (SPIEGEL, 1970; FELFILI \& SILVA JÚNIOR, 1988). Para verificar o crescimento médio anual dos indivíduos no período de quatro anos foi utilizado o modelo apresentado por Imaña-Encinas et al. (2005), conforme Equação 9.

$$
\begin{gathered}
\mathrm{nc}=1+3,3 \times \log \mathrm{n} \\
\mathrm{IC}=\frac{\mathrm{A}}{\mathrm{nc}} \\
\operatorname{IPA}(\mathrm{cm} / \mathrm{ano})=\frac{\sum_{\mathrm{i}=1}^{\mathrm{n}}(\mathrm{DAP} 2 \mathrm{i}-\mathrm{DAP} 1 \mathrm{i})}{\mathrm{N}}
\end{gathered}
$$

Em que: $\mathrm{nc}=$ número de classes; $\mathrm{n}=$ número de indivíduos amostrados; $\mathrm{IC}=$ intervalo de classe; $\mathrm{A}=$ amplitude (maior diâmetro - menor diâmetro); IPA= incremento periódico anual; $\mathrm{DAP} 2 \mathrm{i}$ = diâmetro da iésima árvore viva na segunda medição; DAP1i = diâmetro da iésima árvore viva na primeira medição; e $\mathrm{N}$ = número total de anos monitorado.

\section{RESULTADOS E DISCUSSÃO}

O número de indivíduos apresentou pouca variação durante o período de monitoramento, o qual iniciou com 1091 indivíduos no ano de 2015 (1515 ind/ha) e no ano de 2019 foram mensurados 1064 ind. (1478 ind/ha), nas comunidades avaliadas.

As curvas de rarefação dos períodos comparados indicaram que não houve diferença entre a riqueza nos anos de 2015 e 2019 (Figura 2). Constatou-se que a curva de riqueza observada da comunidade em 2019 (menor comunidade) está dentro do limite de confiança de $95 \%$ da curva de rarefação da comunidade de 2015. No entanto, percebe-se que há uma tendência de pequeno decréscimo de riqueza esperada ao passar do tempo.

Foram encontradas 58 espécies em 2015 a 2017 e 57 espécies nos anos subsequentes. Essa diferença ocorreu pela morte da espécie Albizia niopoides (Spruce ex Benth.) Burkart, Hovenia dulcis Thunb. e Prunus myrtifolia (L.) Urb., representadas por apenas um indivíduo e pelo ingresso de Ilex paraguariensis A.St.-Hil. e uma espécie do gênero Myrtaceae (NI). Em relação a abundância, Actinostemon concolor (Spreng.) Müll.Arg. (211 ind/ha), Gymnanthes klotzschiana Müll.Arg. (139), Sebastiania brasiliensis Spreng. (124), Campomanesia xanthocarpa (Cambess.) O.Berg (118), Matayba elaeagnoides Radlk. (111) e Plinia rivularis (Cambess.) Rotman (104) apresentaram os maiores valores.

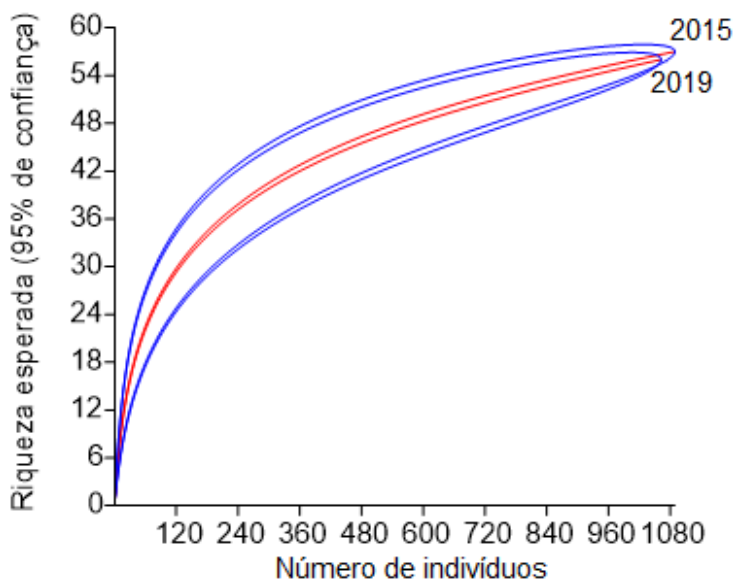

Figura 2. Curvas de rarefação de espécies para a comunidade amostrada para os anos de 2015 e 2019. Guarani das Missões, RS, Brasil. 2020.

Maiores taxas de ocupação do ambiente em área basal foram determinadas para $M$. elaeagnoides $\left(7,67 \mathrm{~m}^{2} / \mathrm{ha}\right), C$. xanthocarpa $(3,41)$, Ruprechtia laxiflora Meisn. $(3,15)$, Luehea divaricata Mart. \& Zucc. $(2,45)$, Cordia americana (L.) Gottschling \& J.S.Mill. $(2,28)$ e Machaerium paraguariense Hassl. (2,14). Com esses resultados verificou-se que essas espécies estão melhor representadas nos fragmentos analisados.

Na primeira avaliação (2015), foram encontradas 15 espécies nativas raras (exemplo: Apuleia leiocarpa (Vogel) J.F.Macbr. e Eugenia burkartiana (D.Legrand) D.Legrand), ou seja, aquelas representadas por apenas um indivíduo amostrado, de acordo com Capers et al. (2005) e Martins (1991). Considerando esse conceito, houve acréscimo no número espécies raras em 2019, quando 16 espécies foram consideraram raras.

Quanto aos grupos ecológicos, observou-se maior proporção de espécies e de indivíduos pertencentes ao grupo clímax exigente de luz, com 36 espécies (683 indivíduos) e 33 espécies (636 indivíduos) em 2015 e 2019 respectivamente, seguidas em ordem decrescente de clímax tolerante à sombra, com 16 espécies (381 e 402 indivíduos) e das pioneiras com 4 espécies (23 e 21 indivíduos). Todavia, quando comparada a proporção de indivíduos dentro de um grupo ecológico no decorrer do tempo não foram encontradas diferenças discrepantes (Tabela 1).

Percebe-se que as espécies clímax dominam ecologicamente as comunidades arbóreas pesquisadas. Esta condição também é evidenciada pelas espécies mais abundantes e de maior área basal, as quais pertenciam aos 
grupos ecológicos clímax tolerante à sombra e clímax exigentes de luz, mostrando que as espécies pioneiras são menos marcantes nesses fragmentos.

Tabela 1. Número de indivíduos e espécies dos grupos ecológicos em cada ocasião de monitoramento. Guarani das Missões, RS, Brasil. 2020.

\begin{tabular}{|c|c|c|c|c|c|c|c|c|}
\hline \multirow{3}{*}{ Grupo } & \multicolumn{5}{|c|}{ Ano de medição } & \multicolumn{3}{|c|}{ Intervalo de tempo } \\
\hline & 2015 & 2016 & 2017 & 2018 & 2019 & Anual & Bienal & Quadr. \\
\hline & \multicolumn{5}{|c|}{ Média de indivíduos por parcela* } & $H$ & $H$ & $H$ \\
\hline Pioneira & $0,6^{c}$ & $0,7^{c}$ & $0,6^{b}$ & $0,7^{b}$ & $0,6^{\mathrm{b}}$ & $0,074^{\text {ns }}$ & $0,059^{\text {ns }}$ & ${ }^{s} 0,041^{\text {ns }}$ \\
\hline CLuz & $19,0^{a}$ & $19,1^{\mathrm{a}}$ & $18,9^{a}$ & $18,3^{\mathrm{a}}$ & $17,7^{a}$ & $0,616^{\mathrm{ns}}$ & $0,488^{\text {ns }}$ & s $0,442^{\text {ns }}$ \\
\hline CSombra & $10,6^{b}$ & $11,3^{b}$ & $11,3^{\mathrm{a}}$ & $11,3^{\mathrm{a}}$ & $11,2^{\mathrm{a}}$ & $0,405^{\text {ns }}$ & $0,280^{\text {ns }}$ & s $0,198^{\text {ns }}$ \\
\hline \multirow[t]{2}{*}{$H$} & $\begin{array}{c}66,714 \\
p<0,01\end{array}$ & $\begin{array}{c}66,857 \\
p<0,01\end{array}$ & $\begin{array}{c}66,412 \\
p<0,01\end{array}$ & $\begin{array}{c}65,772 \\
p<0,01\end{array}$ & $\begin{array}{c}66,3670 \\
p<0,01\end{array}$ & & & \\
\hline & \multicolumn{5}{|c|}{ Média de espécies por parcela* } & $H$ & $H$ & $H$ \\
\hline Pioneira & $0,4^{c}$ & $0,4^{c}$ & $0,4^{c}$ & $0,4^{c}$ & $0,3^{c}$ & $0,105^{\text {ns }}$ & $0,087^{\text {ns }}$ & ${ }^{\mathrm{s}} 0,065^{\text {ns }}$ \\
\hline CLuz & $8,1^{a}$ & $8,0^{a}$ & $8,0^{\mathrm{a}}$ & $7,8 \mathrm{a}$ & $7,7^{\mathrm{a}}$ & $0,401^{\mathrm{ns}}$ & $0,320^{\text {ns }}$ & ${ }^{\mathrm{s}} 0,290^{\text {ns }}$ \\
\hline CSombra & $3,7^{b}$ & $3,8^{b}$ & $3,8^{b}$ & $3,8^{b}$ & $3,8^{b}$ & $0,132^{\text {ns }}$ & $0,060^{\text {ns }}$ & ${ }^{s} 0,047^{\text {ns }}$ \\
\hline$H$ & $\begin{array}{c}75,269 \\
p<0,01\end{array}$ & $\begin{array}{c}76,0,36 \\
p<0,01\end{array}$ & $\begin{array}{c}75,916 \\
p<0,01\end{array}$ & $\begin{array}{c}75,851 \\
p<0,01\end{array}$ & $\begin{array}{c}76,466 \\
p<0,01\end{array}$ & & & \\
\hline
\end{tabular}

* = foram desconsiderados os indivíduos sem classificação do grupo ecológico; CLuz = Clímax Exigente de Luz; CSombra = Clímax tolerante à Sombra. $H$ = estatística do Teste de Kruskal-Wallis; ${ }^{a, b}$ e $\mathrm{c}=$ letras diferentes nas colunas indicam diferença significativa pelo teste de Dunn com p-valor < 0,05; e Grupo Ecol. = Grupo Ecolócico; Quadr.: quadrienal.

De acordo com o teste de Kruskal-Wallis, não há diferença entre a densidade dos grupos ecológicos na comparação entre as medições anuais $(2015,2016,2017$, 2018 e 2019), assim como quando comparadas as populações em biênios (2015, 2017 e 2019) e quadriênios (2015 e 2019). Para que fossem detectadas diferenças de densidade de indivíduos e densidade de espécies entre os grupos ecológicos as parcelas deveriam ser monitoradas por maior período de tempo, ou seja, cinco ocasiões de monitoramento não são suficientes para relatar variações significativas.

No primeiro ano de monitoramento houve maior taxa de recrutamento. Por outro lado, a taxa de mortalidade foi menor nesse mesmo período. Com esses resultados obteve-se taxa de mudança positiva (Tabela 2). Os demais períodos de monitoramento tiveram taxa de mudança negativa, influenciada pelo elevado valor de indivíduos mortos e baixa densidade de recrutamento.

Os maiores valores de mortalidade ocorreram nos anos de 2018 e 2019. Nesse período houve maior incidência de temporais e ventanias na região, causando maior tombamento de árvores no interior da floresta, o que influenciou diretamente no valor da taxa de mudança nesse período (TMD= $-4,7$ no período 2017 a 2019). As taxas de mortalidade elevadas tendem a influenciar as taxas de recrutamento em períodos futuros, visto que, a abertura de clareiras facilita a entrada e crescimento de novas espécies ou indivíduos (CORRÊA \& SANTOS, 2015).

Tabela 2. Parâmetros de dinâmica em fragmentos de Floresta Estacional Decidual. Guarani das Missões, RS.

\begin{tabular}{|c|c|c|c|c|c|c|c|}
\hline \multirow{3}{*}{ Parâmetros } & \multicolumn{7}{|c|}{ Intervalo de tempo } \\
\hline & \multicolumn{4}{|c|}{1 ano } & \multicolumn{2}{|c|}{2 anos } & 4 anos \\
\hline & $15-16$ & $16-17$ & $17-18$ & $18-19$ & $15-17$ & $17-19$ & $15-19$ \\
\hline \multicolumn{8}{|c|}{ Número de árvores } \\
\hline Inicial (ind./ha) & 1515,3 & 1558,3 & 1550,0 & 1519,4 & 1515,3 & 1550,0 & 1515,3 \\
\hline \multicolumn{8}{|l|}{ Sobreviventes (ind./ha)1 } \\
\hline Recrutas (ind./ha) & 59,7 & 18,1 & 23,6 & 19,4 & 75,0 & 34,7 & 34,7 \\
\hline Mortos (ind./ha) & 16,7 & 23,6 & 54,2 & 61,1 & 40,3 & 106,9 & 136,1 \\
\hline Final (ind./ha) & 1558,3 & 1550,0 & 1519,4 & $\$ 1477,8$ & 31550,0 & 1477,8 & 1477,8 \\
\hline Tx mudança (\%) & 2,8 & $-0,5$ & $-2,0$ & $-2,7$ & 2,3 & $-4,7$ & $-2,5$ \\
\hline Tx recrutam. (\%) & 3,8 & 1,2 & 1,6 & 1,3 & 4,8 & 2,3 & 2,3 \\
\hline Tx mortalidade (\%) & 1,1 & 1,5 & 3,5 & 4,0 & 2,7 & 6,9 & 9,0 \\
\hline \multicolumn{8}{|c|}{ Área basal } \\
\hline Inicial (m²/ha) & 34,94 & 35,985 & 36,199 & 35,968 & 334,941 & 36,199 & 34,941 \\
\hline Aumento $\left(\mathrm{m}^{2} / \mathrm{ha}\right)$ & 1,365 & 0,855 & 1,003 & 0,935 & 2,079 & 1,761 & 3,610 \\
\hline Decréscimo $\left(\mathrm{m}^{2} / \mathrm{ha}\right)$ & 0,260 & 0,184 & 0,323 & 0,136 & 0,315 & 0,237 & 0,357 \\
\hline Recrutas (m²/ha) & 0,242 & 0,064 & 0,103 & 0,104 & 0,310 & 0,129 & 0,369 \\
\hline Mortos ( $\left.\mathrm{m}^{2} / \mathrm{ha}\right)$ & 0,302 & 0,522 & 1,014 & 1,042 & 0,815 & 2,023 & 2,735 \\
\hline Final $\left(\mathrm{m}^{2} / \mathrm{ha}\right)$ & 35,985 & 36,199 & 35,968 & 335,828 & 336,199 & 35,828 & 35,828 \\
\hline Taxa mudança (\%) & 3,0 & 0,6 & $-0,6$ & $-0,4$ & 3,6 & $-1,0$ & 2,5 \\
\hline Perda (\%) & 1,6 & 2,0 & 3,7 & 3,3 & 3,2 & 6,2 & 8,8 \\
\hline Ganho (\%) & 4,5 & 2,5 & 3,1 & 2,9 & 6,6 & 5,3 & 11,1 \\
\hline
\end{tabular}

Em relação a área basal, as taxas de mudança e taxa de ganho tiveram valores mais expressivos no período 20152016. Esses resultados decorreram principalmente do crescimento das árvores sobreviventes e do ingresso de novos indivíduos.

Os resultados da distribuição diamétrica (Figura 3) indicam pequenas alterações de densidade em algumas classes de diâmetro em nível de comunidade, sendo mais evidente a diferença de densidade entre as árvores de menor porte $(5 \mathrm{~cm} \leq$ diâmetro $<12 \mathrm{~cm}$ ). No geral, a vegetação apresentou concentração de maior número de indivíduos nas classes iniciais, com curva em forma exponencial negativa, demonstrando capacidade autorregenerativa e de manutenção dos níveis atuais de densidade (NASCIMENTO et al., 2004). 


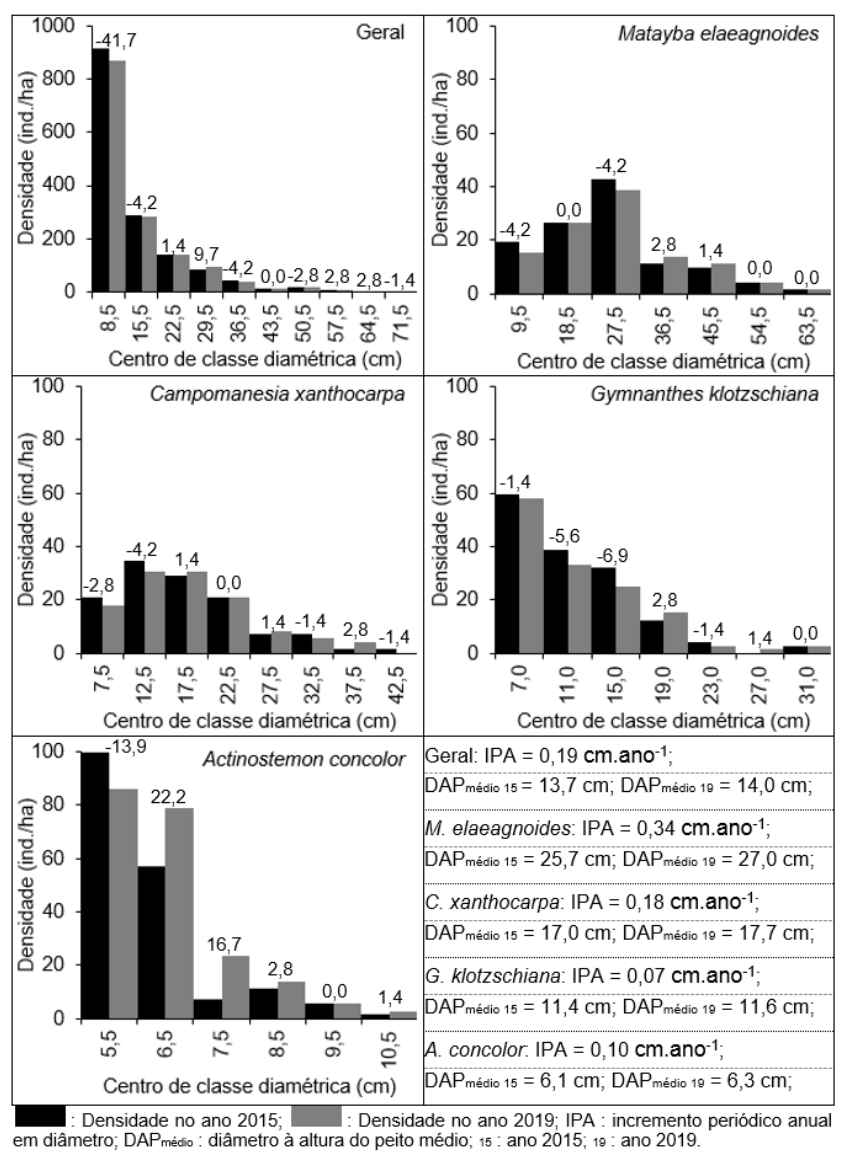

Figura 3. Densidade de indivíduos por classes diamétricas.

Padrão similar foi observado para Gymnanthes klotzschiana que teve menor variação nas classes de menor diâmetro e apresentou padrão de distribuição semelhante ao da comunidade. Machado et al. (2010) relataram padrão similar para essa espécie em Floresta Estacional Semidecidual em Minas Gerais, onde o maior número de indivíduos da espécie pertenceu às menores classes diamétricas. Em contraste, Matayba elaeagnoides teve tendência a distribuição normal e maior variação de densidade em classes intermediárias, com perda de indivíduos nas classes iniciais e aumento da densidade nas classes de diâmetros maiores, ou seja, houve mortalidade e/ou progressão de classe de alguns indivíduos.

Campomanesia xanthocarpa teve maior flutuação na primeira e na segunda classe diamétrica, resultado que pode estar associado a relação da espécie com diversos usos nas propriedades rurais, já que a espécie, segundo Sauersig (2014), possui madeira resistente usada para carpintaria, lenha e carvão, além do uso medicinal, alimentício e ornamental. Através do padrão gerado para esta espécie e para Matayba elaeagnoides, verificou-se que ambas tendem a apresentar problemas para a renovação do estrato arbóreo futuramente, pois tiveram poucos indivíduos na primeira classe de diâmetro. Silva et al. (2012) também verificaram padrão de distribuição descontínuo, com maior número de indivíduos nas maiores classes de diâmetro para a espécie $M$. elaeagnoides, a qual foi a quarta espécie com maior VI estudado pelo elevado valor de dominância, já que a espécie apresentou poucos indivíduos. Em geral, as espécies analisadas sofreram pequenas oscilações de densidade, sendo essas associadas às classes diamétricas com maior concentração de indivíduos.

Actinostemon concolor, por suas características morfométricas teve diâmetro máximo de $10,7 \mathrm{~cm}$, o que justifica a ocorrência dessa espécie apenas na primeira classe da distribuição geral, contribuindo para a elevada densidade de indivíduos desta classe. A. concolor teve padrão de distribuição diamétrica próximo ao da floresta em geral (J-invertido) e se manteve entre as espécies de maior valor de cobertura pela sua elevada densidade, já que apresentou baixa dominância no presente estudo.

De forma geral, a comunidade apresentou incremento periódico anual (IPA) de 0,19 cm.ano-1. Em trecho de Floresta Estacional Semidecidual, MT, Mews et al. (2011) encontraram resultados aproximados (IPA=0,15 cm.ano-1), em período de monitoramento de cinco anos. As espécies com maior valor de cobertura tiveram crescimento distinto uma das outras. $M$. elaeagnoides teve maior incremento em diâmetro (IPA=0,34 cm.ano ${ }^{-1}$ ), contrastando com $G$. klotzschiana que teve menor IPA das espécies analisadas $\left(0,07 \mathrm{~cm} . a n o^{-1}\right)$, Kanieski (2013) mostrou que essa última espécie tem crescimento muito baixo, apresentando pouca variação em diâmetro.

\section{CONCLUSÕES}

Não foram detectadas diferenças significativas em relação a riqueza de espécies no período monitorado. Espécies clímax dominam ecologicamente os fragmentos do presente estudo e plantas classificadas no grupo ecológico clímax exigente de luz tiveram maior representatividade em número de espécie e de indivíduos. No entanto não houve variação nos períodos estudados, o que sugere maior tempo de observação para que sejam detectadas diferenças.

Maiores taxas de mudança foram registradas nos anos iniciais de monitoramento. Através da análise de distribuição diamétrica pode-se concluir que a comunidade analisada como um todo é capaz de manter a estrutura atual no futuro próximo, exceto quando analisada a estrutura para algumas espécies de maneira isolada, como para Matayba elaeagnoides e Campomanesia xanthocarpa que tendem a apresentar problemas de renovação na 
floresta, pela ausência de estoque de indivíduos nas classes de menor diâmetro.

\section{REFERÊNCIAS}

AYRES, M. et al. BioEstat: aplicações estatísticas nas áreas das ciências biomédicas. Belém: Instituto de Desenvolvimento Sustentável Mamirauá, 2007.

ALVARES, C.A. et al. Köppen's climate classification map for Brazil. Meteorologische Zeitschrift, v.22, n.6, p.711-728, 2013.

ANDRZEJEWSKI, C. Estrutura e florística de dois fragmentos ripários de Floresta Estacional Decidual no Noroeste do Rio Grande do Sul, Brasil. 2016. 8p. (Dissertação de Mestrado).

BYNG, J.W. et al. An update of the angiosperm phylogeny group classification for the orders and families of flowering plants: APG IV. Botanical Journal of the Linnean Society, v.181, n.1, p.1-20, 2016.

CALLEGARO, R.M.; ARAUJO, M.M.; LONGHI S.J. Fitossociologia de agrupamentos em Floresta Estacional Decidual no Parque Estadual Quarta Colônia, Agudo-RS. Revista Brasileira de Ciências Agrárias, v.9, n.4, p.590-598, 2014.

CAPERS. R.S. et al. Successional dynamics of woody seedling communities in wet tropical secondary forests. Journal of Ecology, v.93, p.1071-1084, 2005.

CORRÊA, C. E.; SANTOS, F. A. M. Padrões em comunidades podem ser entendidos pelo comportamento das populações que as compõem? Interface entre estudos de populações e comunidades. Fitossociologia no Brasil: métodos e estudos de casos. v. 2. Viçosa: UFV, 2015. cap. 13, p.288-301.

FELFILI, J.M.; REZENDE, R.P. Conceitos e métodos em fitossociologia. Brasília: Universidade de Brasília, 2003.

FELFILI, J.M.; SILVA JÚNIOR, M.C. Distribuição dos diâmetros numa faixa de Cerrado da Fazenda Água Limpa (FAL) em Brasília. Acta Botanica Brasilica, v.2, n.1-2, p.85-104, 1988.

FONTES, C.G.; WALTER, B.M.T. Dinâmica do componente arbóreo de uma mata de galeria inundável (Brasília, Distrito Federal) em um período de oito anos. Revista Brasileira de Botânica, v.34, n.2, p.145-158, 2011.

FORZZA, R.C. et al. Flora do Brasil 2020. Jardim Botânico do Rio de Janeiro. Disponível em: www.floradobrasil.jbrj.gov.br

GUNDERSEN, P. et al. Environmental services provided from riparian forests in the nordic countries. Journal of the Human Environment, v.39, n.8, p.555-566, 2010.

HAMMER, O.; HARPER, D.A.T.; RYAN, P.D. PAST: Paleontological statistics software package for education and data analysis. Palaentologia Electronica, v.4, n.1, p.1-9, 2001.

HIGUCHI, P. et al. Spatio-temporal patterns of tree community dynamics in a tropical forest fragment in South-east Brazil. Plant Ecology, v.199, n.1, p.125-135, 2008.
IBGE - INSTITUTO BRASILEIRO DE GEOGRAFIA E ESTATÍSTICA. Manual técnico da vegetação brasileira: sistema fitogeográfico; inventário das formações florestais e campestres; técnicas e manejo de coleções botânicas; procedimentos para mapeamentos. 2. ed. Rio de Janeiro: IBGE, 2012.

IMAÑA-ENCINAS, J.; SILVA, G.F.; PINTO, J.R.R. Idade e crescimento das árvores. Brasília: UNB, 2005.

KANIESKI, M.R. Estudo dendroecológico de Sebastiania commersoniana (Baill.) L.B.Sm. \& R.J.Downs e de arbóreas invasoras em Floresta Ombrófila Mista aluvial, Sul do Brasil. 2013. 95p. (Tese de Doutorado).

KORNING, J.; BALSLEV, H. Growth and mortality of trees in Amazonian tropical rain forest in Ecuador. Journal of Vegetation Science, v.4, n.1, p.77-86, 1994.

MACHADO, E.L.M. et al. Flutuações temporais nos padrões de distribuição diamétrica da comunidade arbóreo-arbustivo e de 15 populações em um fragmento florestal. Revista Árvore, v.34, n.4, p723-732, 2010.

MARTINS, F.R. Estrutura de uma floresta mesófila. Campinas: Editora da UNICAMP, 1991.

MEWS, H.A. et al. Dinâmica estrutural da comunidade lenhosa em Floresta Estacional Semidecidual na transição Cerrado-Floresta Amazônica, Mato Grosso, Brasil. Acta Botanica Brasilica, v.25, n.4, p.845-857, 2011.

NAIMAN, R.J.; DÉCAMPS, H. The ecology of interfaces: riparian zones. Annual Review of Ecology and Systematics, v.28, n.1, p.621-658, 1997.

NASCIMENTO, A.R.T.; FELFILI, J.M.; MEIRELLES, E.M. Florística e estrutura da comunidade arbórea de um remanescente de Floresta Estacional Decidual de encosta, Monte Alegre, GO, Brasil. Acta Botanica Brasilica, v.18, n.3, p.659-669, 2004.

OLIVEIRA-FILHO, A.T. et al. Effects of soils and topography on the distribution of tree species in a tropical riverine forest in southeastern Brazil. Journal of Tropical Ecology, v.10, n.4, p.483-508, 1994.

OLIVEIRA, A.P. et al. Mortality, recruitment and growth of the tree communities in three forest formations at the Panga Ecological Station over ten years (1997-2007). Acta Botanica Brasilica, v.28, n.2, p.234-248, 2014.

RODRIGUES, R.R.; GANDOLFI, S. Conceitos, tendências e ações para a recuperação de florestas ciliares. Matas ciliares: conservação e recuperação. São Paulo: EDUSP, 2004. p.235-247.

SARAIVA, D.D. Composição e estrutura de uma floresta ribeirinha no sul do Brasil. Biotemas, v.24, n.4, p.49-58, 2011.

SAUERSIG, D. Plantas do Brasil: árvores nativas. Irati: Editora Plantas do Brasil, 2014.

SHEIL, D.; MAY, R.M. Mortality and recruitment rate evaluations in heterogeneous tropical forests. Journal of Ecology, v.84, n.1, p.91-100, 1996. 
SILVA, A.C. et al. Relações florísticas e fitossociologia de uma Floresta Ombrófila Mista Montana secundária em Lages, Santa Catarina. Ciência Florestal, v.22, n.1, p.193-206, 2012.

SPIEGEL, M.R. Estadística: teoría y 875 problemas resueltos. Mexico: McGraw-Hill, 1970.

STRECK, E.V. et al. Solos do Rio Grande do Sul. Porto Alegre: EMATER-RS, 2008.

SWAINE, M.D.; WHITMORE, T.C. On the definition of ecological species groups in tropical rain forests. Vegetatio, v.75, n.1-2, p.81-86, 1988.

VENZKE, T.S.; MARTINS, S.V. Aspectos florísticos de três estágios sucessionais em mata ciliar em Arroio do Padre, extremo sul do Brasil. Floresta, v.43, n.2, p.191-204, 2013.

VIANA, V.M.; PINHEIRO, L.A.F.V. Conservação da biodiversidade em fragmentos florestais. Série Técnica IPEF, v.12, n.32, p. 25-42, 1998.

WREGE, M.S. et al. Atlas climático do Rio Grande do Sul. Porto Alegre: CEMET/FEPAGRO, 2011.

Recebido em 21-02-2020

Aceito em 22-04-2020 